\title{
Gamma-Ray Bursts Polarization
}

\author{
Diego Götz ${ }^{1}$ and Stefano Covino ${ }^{2}$ \\ ${ }^{1}$ CEA Saclay - DRF/Irfu/Service d'Astrophysique, \\ Orme des Merisiers, Bât. 709, F-91191 Gif-sur-Yvette, France \\ email: diego.gotz@cea.fr \\ ${ }^{2}$ INAF / Brera Astronomical Observatory, \\ Via Bianchi 46, 23907, Merate (LC), Italy \\ email: stefano.covino@brera.inaf.it
}

\begin{abstract}
We review the current observational and theoretical status of the polarization measurements of Gamma-ray Bursts at all wavelengths. Gamma-Ray Bursts are thought to be produced by an ultra-relativistic jet, possibly powered by a black hole. One of the most important open point is the composition of the jet: the energy may be carried out from the central source either as kinetic energy (of baryons and/or pairs), or in electromagnetic form (Poynting flux). The polarization properties are expected to help disentangling main energy carrier. The prompt emission and afterglow polarization are also a powerful diagnostic of the jet geometry.
\end{abstract}

Keywords. Polarization, gamma rays: bursts, gamma rays: observations, techniques: photometric

\section{Introduction}

Polarimetric measurements can provide useful complementary (wrt. timing and spectroscopy) information about the physical processes at work in Gamma-Ray Bursts (GRBs). Indeed several different possible scenarios have been invoked to interpret the large amount of observational data now available for GRBs. Most of the theoretical efforts have been applied to the so-called "standard model", which, although a fully satisfactory picture is still missing, offers the best interpretative scenario for the polarimetric observations.

In this paper we first summarize the observational status of the GRB prompt emission polarimetric measurements, and then compare them to theoretical predictions. Then we will do the same concerning the GRB afterglow phase, and we conclude with some perspectives on the domain. For more details on GRB polarimetric measurements and their implications also on fundamental physics, see the recent review by Covino \& Götz (2016).

\section{Polarization in the prompt phase}

The measurement of polarization during the prompt phase of GRBs has always been challenging. This is mainly due to the fact that no wide field gamma-ray polarimeter with a large effective area has yet been flown, and that many of the measurements attempted to date have been performed with instruments which have some polarimetric capabilities, but have not an explicit polarimetric oriented design. In addition at odd to the afterglow emission, the GRB prompt emission is very limited in time, mostly less than a few hundreds of seconds, and hence, in spite of the high average flux of GRBs, the total number of collected photons is often too limited to derive statistically stringent limits for polarization. 


\subsection{Early Results}

The first attempt to measure linear polarization in the prompt emission of GRBs was reported by Coburn \& Boggs (2003). They used the Reuven Ramaty High Energy Solar Spectroscopic Imager (RHESSI) observations of GRB 021206. RHESSI has been designed to study solar flares in the $3 \mathrm{keV}-17 \mathrm{MeV}$ energy range. In the soft gamma-ray energy range $(0.15-2.0 \mathrm{MeV})$ the dominant photon interaction in RHESSI is Compton scattering. Polarization at high energies can be measured, thanks to the polarization dependency of the differential cross section for Compton scattering

$$
\frac{d \sigma}{d \Omega}=\frac{r_{0}^{2}}{2}\left(\frac{E^{\prime}}{E_{0}}\right)^{2}\left(\frac{E^{\prime}}{E_{0}}+\frac{E_{0}}{E^{\prime}}-2 \sin ^{2} \theta \cos ^{2} \phi\right)
$$

where $r_{0}^{2}$ is the classical electron radius, $E_{0}$ the energy of the incident photon, $E^{\prime}$ the energy of the scattered photon, $\theta$ the scattering angle, and $\phi$ the azimuthal angle relative to the polarization direction. Linearly polarized photons scatter preferentially perpendicularly to the incident polarization vector. Hence by examining the angles of scattering of the photons among the different RHESSI detectors, one can in principle derive the degree and angle of linear polarization of the incident photons.

Coburn \& Boggs (2003) reported a high level of linear polarization of $\Pi=80 \pm 20 \%$ (close to and beyond the theoretical value) at a high level of confidence $(>5.7 \sigma)$ for GRB 021206. GRB 021206 was a quite bright GRB with a fluence of $1.6 \times 10^{-4} \mathrm{erg}$ $\mathrm{cm}^{-2}$ in the 25-100 keV energy band, and a peak flux of $2.9 \times 10^{-5} \mathrm{erg} \mathrm{cm}^{-2} \mathrm{~s}^{-1}$. Coburn \& Boggs (2003) interpreted the angular modulation measured in the data as a high-level polarization signal. However subsequent re-analyses of the same data set could not confirm this result reporting a polarization level compatible with zero (Rutledge et al. 2004; Wigger et al. 2004). These authors show that the number of suitable events for polarization analysis has been over-estimated by a factor 10 (830 150 versus $9840 \pm 96)$, since spurious coincidences had been counted as Compton scattering events, implying in the end a too small statistics for being able to measure any polarization signal, even for a $100 \%$ polarized source.

\subsection{IBIS and SPI on board INTEGRAL}

At the time of its discovery GRB 041219A was among the top 1\% in terms of GRB fluence. This prompted different attempts to measure its polarization with the instruments that observed it. The first attempt was performed by McGlynn et al. (2007) using the SPI spectrometer on board INTEGRAL. They were able to measure the degree of linear polarization over the brightest pulse of the GRB (lasting $66 \mathrm{~s}$ ) to $\Pi=63_{-30}^{+31} \%$ and the polarization angle to $P A=70_{11}^{+14}$ degrees. GRB041219A was also observed by the Imager on Board the INTEGRAL Satellite (IBIS). Thanks to its two superposed pixellated detection planes, IBIS can be used as a Compton Polarimeter. By examining the scatter angle distribution of the detected photons in the two planes

$$
N(\phi)=S\left[1+a_{0} \cos 2\left(\phi-\phi_{0}\right)\right]
$$

one can derive the polarization angle, $P A=\phi_{0}-\pi / 2+n \pi$, and the polarization fraction $\Pi=a_{0} / a_{100}$, where $a_{100}$ is the amplitude expected for a $100 \%$ polarized source derived by Monte Carlo simulations. The IBIS results are reported in Table 1.

Using the same method polarization could be measured for two other GRBs $(061122 \dagger$ and 140206A) with IBIS by Götz et al. (2013), Götz et al. (2014), see Table 2, but no

$\dagger$ A consistent polarization measurement has been obtained with SPI. 
Table 1. Polarization results for GRB 041219A for the different time intervals. From Götz et al. (2009)

\begin{tabular}{cccccc}
\hline Name & $\begin{array}{c}\mathrm{T}_{\text {start }} \\
\text { U.T. }\end{array}$ & $\begin{array}{c}\mathrm{T}_{\text {stop }} \\
\text { U.T. }\end{array}$ & $\begin{array}{c}\Pi \\
\%\end{array}$ & $\begin{array}{c}P A \\
\text { degrees }\end{array}$ & $\begin{array}{c}\text { Image } \\
\text { SNR }\end{array}$ \\
\hline First Peak & $01: 46: 22$ & $01: 47: 40$ & $<4$ & - & 32.0 \\
Second Peak & $01: 48: 12$ & $01: 48: 52$ & $43 \pm 25$ & $38 \pm 16$ & 20.0 \\
P6 & $01: 46: 47$ & $01: 46: 57$ & $22 \pm 13$ & $121 \pm 17$ & 21.5 \\
P8 & $01: 46: 57$ & $01: 27: 07$ & $65 \pm 26$ & $88 \pm 12$ & 15.9 \\
P9 & $01: 47: 02$ & $01: 47: 12$ & $61 \pm 25$ & $105 \pm 18$ & 18.2 \\
P28 & $01: 48: 37$ & $01: 48: 47$ & $42 \pm 42$ & $106 \pm 37$ & 9.9 \\
P30 & $01: 48: 47$ & $01: 48: 57$ & $90 \pm 36$ & $54 \pm 11$ & 11.8 \\
\hline
\end{tabular}

Errors are given at $1 \sigma$ c.l. for one parameter of interest.

Table 2. Summary of recent GRB polarization measurement by IBIS/SPI and GAP.

\begin{tabular}{ccccccc}
\hline GRB & $\begin{array}{c}\Pi \\
(68 \% \text { c.l. })\end{array}$ & $\begin{array}{c}\text { Peak energy } \\
(\mathrm{keV})\end{array}$ & $\begin{array}{c}\text { Fluence } \\
\left(\mathrm{erg} \mathrm{cm}^{-2}\right)\end{array}$ & Energy Range & $\begin{array}{c}\text { Redshift } \\
z\end{array}$ & Instrument \\
\hline $041291 \mathrm{~A}$ & $65 \pm 26 \%$ & $201_{-41}^{+80}$ & $2.5 \times 10^{-4}$ & $20-200 \mathrm{keV}$ & $0.31_{-0.26}^{+0.54}$ & IBIS, SPI \\
06122 & $>60 \%$ & $188 \pm 17$ & $2.0 \times 10^{-5}$ & $20-200 \mathrm{keV}$ & $1.33_{-0.76}^{+0.77}$ & IBIS, SPI \\
$100826 \mathrm{~A}$ & $27 \pm 11 \%$ & $606_{-109}^{+134}$ & $3.0 \times 10^{-4}$ & $20 \mathrm{keV}-10 \mathrm{MeV}$ & $0.71-6.84^{1}$ & GAP \\
$110301 \mathrm{~A}$ & $70 \pm 22 \%$ & $107 \pm 2$ & $3.6 \times 10^{-5}$ & $10 \mathrm{keV}-1 \mathrm{MeV}$ & $0.21-1.09^{1}$ & GAP \\
110721 & $84_{-28}^{+16} \%$ & $393_{-104}^{+199}$ & $3.5 \times 10^{-4}$ & $10 \mathrm{keV}-1 \mathrm{MeV}$ & $0.45-3.12^{1}$ & GAP \\
$140206 \mathrm{~A}$ & $>48 \%$ & $98 \pm 17$ & $2.0 \times 10^{-5}$ & $15-350 \mathrm{keV}$ & $2.739 \pm 0.001$ & IBIS \\
\hline
\end{tabular}

${ }^{1}$ redshift based on empirical prompt emission correlations, not on afterglow observations.

time-resolved analysis could be performed due to the limited statistics, making GRB041219A the only GRB for which a time variable polarization signal could be measured to date with IBIS.

\section{3. $G A P$}

High levels of linear polarization could be measured also for three GRBs (100826A, 110301A and 110721) by the Gamma-Ray Burst Polarimeter experiment on board the IKAROS spacecraft (Yonetoku et al. 2011 \& 2012). In particular for GRB 100826A, which had a similarly high fluence as 041219A, Yonetoku et al. (2011) were able to measure a change in the polarization angle by dividing the GRB in two $\sim 50 \mathrm{~s}$ long time intervals: the angle changed from $159 \pm 18^{\circ}$ to $75 \pm 20^{\circ}$ ( $1 \sigma$ c.l. for two parameters of interest) with a significance of $3.5 \sigma$ for the change.

\subsection{Interpretation}

The expected level of polarization of the prompt $\gamma$-ray emission in GRBs has been estimated by several authors for different models, or variations within them. In most cases, the observed $\gamma$-ray emission is due to the synchrotron radiation from relativistic electrons. To have a high radiative efficiency and to allow for the short time scale variability in the GRB light curves, these electrons have to be in the fast cooling regime. The intrinsic polarization level of the synchrotron radiation (assuming a time-averaged electron distribution $n(\gamma)=\gamma^{-p}$ with $p^{\prime}=p+1$ above $\Gamma_{m}$ and $p^{\prime}=2$ below $) \Pi_{\text {syn }}=\left(p^{\prime}+1\right) /\left(p^{\prime}+7 / 3\right)$ is then of the order of $\Pi_{\text {syn }}=(p+2) /(p+10 / 3) \simeq 75 \%$ above $\nu_{\mathrm{m}}$ and $\Pi_{\text {syn }}=9 / 12 \simeq 70 \%$ below, where $\nu_{\mathrm{m}}$, the peak of the spectrum in $\nu F_{\nu}$, is the synchrotron frequency of electrons at $\Gamma_{\mathrm{m}}$ (the minimum Lorentz factor of the injected electrons). High polarization 
levels can also be reached if inverse Compton scatterings are the dominant radiative process.

Actually different scenarios in terms of radiation processes and observer's viewing angle can be envisaged to explain the presence of polarized emission during the prompt phase of GRB emission. They can be roughly divided in two families: intrinsic models and geometric models, for which peculiar observing conditions are required.

(a) Synchrotron emission from shock-accelerated electrons in a relativistic jet with an ordered magnetic field contained in the plane perpendicular to the jet expansion. This scenario leads to a maximum time-averaged polarization in long intervals of $\Pi / \Pi_{\mathrm{max}} \sim$ 0.6, i.e. $\Pi \sim 45 \%$ in this case (Granot 2003, Granot \& Königl 2003, Nakar et al. 2003). The main requirement for this model to apply is to have a uniform magnetic field in space, i.e. with a coherence spatial scale $R \theta_{B}$ with $\theta_{B} \gtrsim 1 / \Gamma$. If $\theta_{B}$ is smaller, so that a number $N \sim\left(\Gamma \theta_{B}\right)^{-2}$ of mutually incoherent patches are present in the visible region, the level of polarization will decrease, but the variability (both of the polarization level and angle) will increase (Granot 2003). Indeed if the radiating electrons are accelerated in internal shocks, the Lorentz factor associated with the individual shells is necessarily varying in the outflow, which can be an additional source of variability for the polarization. This scenario could hence produce time variable polarization, as long as the coherence scale $\theta_{\mathrm{B}}$ of the field is larger than $1 / \Gamma$ in most of the emitting regions.

(b) Synchrotron emission from a purely electromagnetic outflow. In this scenario the GRB is powered by the rotational energy of a magnetar-like progenitor (e.g. Metzger et al. 2011). The estimated level of polarization in this case is comparable with the previous scenario (up to $~ 50 \%$ ) (Lyutikov et al. 2003). In addition, a magnetic field with a large coherence scale is naturally expected in such a purely electromagnetic outflow.

(c) Synchrotron emission from shock-accelerated electrons in a relativistic jet with a random field generated at the shock and contained in the plane perpendicular to the jet velocity. A high level of polarization can be obtained even with a random magnetic field if the jet is observed from just outside its edge (Ghisellini \& Lazzati 1999, Waxman 2003). The polarization at the peak of a given pulse can reach $\Pi / \Pi_{\text {syn }} \simeq 0.8$, i.e. $\Pi \simeq 60 \%$ resulting in a time-integrated value of the order of $\Pi / \Pi_{\text {syn }} \simeq 0.5-0.6$, i.e. $\Pi \simeq 40-45 \%$ (Granot 2003, Granot \& Königl 2003, Nakar et al. 2003). However these high values are obtained if the jet is seen with $\theta_{\mathrm{obs}} \simeq \theta_{\mathrm{j}}+1 / \Gamma$, where $\theta_{\mathrm{j}}$ is the opening angle of the jet and $\theta_{\mathrm{obs}}$ the angle between the line-of-sight and the jet axis. Such viewing conditions are rare, except if $\theta_{\mathrm{j}} \sim 1 / \Gamma$.

(d) Synchrotron emission from shock-accelerated electrons in a relativistic jet with an ordered magnetic field parallel to the jet velocity. This case has been studied by Granot \& Königl 2003 and gives very similar results to the previous model. The viewing conditions have to be the same and it suffers the same difficulties as listed above;

(e) Inverse Compton emission from relativistic electrons in a jet propagating within a photon field ("Compton drag" model). The level of polarization in this scenario can be even higher than for the synchrotron radiation and reach $60-100 \%$, but only under the condition that the jet is narrow with $\Gamma \theta_{j} \lesssim 5$, see Lazzati et al. (2004). The maximum level of polarization is again obtained for $\theta_{o b s} \simeq \theta_{j}+1 / \Gamma$. These viewing conditions are very similar to those of model (c). Again, the polarization is reduced if the edges of the jet are not sharp enough. Variability of the Lorentz factor will again result in a varying polarization, with the same difficulties regarding the final level of polarization than in model (c). However, variations of the Lorentz factor could possibly be less large in this scenario as part of the variability of the light curve can be related to the inhomogeneity of the ambient photon field. 
(f) Independently from the emission process (synchrotron or inverse Compton), fragmented fireballs (shotguns, cannonballs, sub-jets) can produce highly polarized emission, with a variable polarization amplitude. The fragments are responsible for the single pulses and have different intrinsic properties (such as Lorentz factors), opening angles, orientations with respect to the observers and magnetic domains. (e.g. Lazzati \& Begelman 2009). In this case the most polarized pulses are those which have about one tenth of the flux of the main pulse, i.e. an anti-correlation between the polarization level and the GRB pulse flux is expected.

\section{Afterglow emission}

Due to the large data set of polarimetric observation during the GRB afterglow phase we address the reader to the Covino \& Götz (2016) paper for a complete picture, and here we restrict ourselves only to the most relevant measurements.

\subsection{GRB 990510}

Polarimetry at a few percent level can be demanding for rapidly fading sources as GRB afterglows, and therefore is not a surprise that the first positive detections in the optical band came a few months after the first unit of the VLT, with its collecting area and flexibility, become operational. GRB 990510 was observed two times by two independent teams (Covino et al. 1999, Wijers et al. 1999) at 18-21 hours after the burst with the ESO-VLT, providing a small but highly significant polarization level at $P=1.7 \pm 0.2 \%$. A later measurement one day after gave a result consistent with a non-variability of the observed polarization.

Polarization at this level is not common for extragalactic sources, however it is possible that it is due to polarization induced by dust grains interposed along the line of sight, which may be preferentially aligned due to the galactic magnetic fields. Large variations are anyway expected, and observed, for specific line of sights. On the other hand, the effect due to dust in the Galaxy can be in principle easily checked and removed if a sufficiently large number of stars are observed in the same field of view. Electron scattering can also lead to some polarization, as observed in supernove and usually attributed to asymmetries in their photospheres (Wang \& Wheeler 2008). However, the degree of induced polarization is of the order of the electron optical depth that cannot be more than $\sim 10^{-6}$ a day after the event (Wijers et al. 1999).

GRB 990510 was also the first GRB with an achromatic (at least in the optical band) steepening, a jet-break, of the afterglow light-curve clearly observed (Israel et al. 1999, Harrison et al. 1999, Stanek et al. 1999). The observations with solid polarization detections were performed before the jet-break. Due to the large Lorentz factor of the outflow, $\Gamma$, only a fraction $\sim 1 / \Gamma$ of the emitting region is accessible to the observer. Photons produced in regions at an angle $1 / \Gamma$ with respect to the line of sight are emitted, in the comoving frame, at $\sim 90^{\circ}$ from the velocity vector. A comoving observer at this angle can then see a compressed emitting region (Ghisellini \& Lazzati 1999) and a projected magnetic field structure with a preferred orientation. If the gradual steepening of the light curve is a jet-break, we would observe only regions at a viewing angle $1 / \Gamma$ at variance with an axis-symmetric situation, and this asymmetry can be the cause of the observed linear polarization that therefore becomes the "smoking gun" of synchrotron emission for GRB afterglows. 


\subsection{Time evolution measurements}

The attempt to identify a time-evolution of the polarization degree and position angle generated a richer dataset for several events. GRB 020405 was observed with the VLA by Granot \& Taylor 2005, the VLT by Masetti et al. (2003) and Covino et al. (2003) and the Multiple Mirror Telescope by Bersier et al. (2003) between one and three days from the burst. The polarization level was observed at about $1.2-2 \%$ for the VLT observations but at the MMT a much higher polarization at about $10 \%$ was detected. Only mild upper limits were obtained at the radio frequencies. The position angle possibly showed a slow change $\left(\sim 10^{\circ}\right)$ from the first to the last observations. The observations derived with the VLT before and after the MMT observations are substantially consistent with a constant afterglow polarization, possibly also with an important contribution of dust in the host galaxy. The rapid variation required to move from the $\sim 1 \%$ to $\sim 10 \%$ in a timescale of about one hour is essentially inconsistent with basically all the geometric models and also with the patchy-shell idea (Ghisellini \& Lazzati 1999, Sari 1999, Gruzinov \& Waxman 1999). In principle a micro-lensing phenomenon Loeb \& Perna (1998) could be responsible for the polarization "flare", although the rapid time scale, the almost constant position angle and the lack of an analogous brightening in the total flux curve make even this interpretation unlikely. The high polarization observed by Bersier et al. (2003) only about one hour after the observation carried out by Masetti et al. (2003) is therefore still of difficult interpretation.

\subsection{GRB 030329}

A fundamental breakthrough in the observational activities of GRB afterglows occurred with GRB 030329 (see Covino \& Götz (2016) for a complete set of references). GRB 030329 was discovered by the HETEII satellite and was one of the few cases of low redshift GRBs $(z \sim 0.17)$. Being in addition a regular cosmological GRB, i.e. not part of the category of low-luminosity low-redshift events, it showed an optical brightness sufficiently high to allow about one month of uninterrupted polarimetric observations in the optical with the ESO-VLT, the CAHA, the NOT, the IAG-USP and much longer in the radio with the VLBA. The afterglow polarization showed a strong variability in polarization degree and position angle. The polarization was typically in the $0.3-2.5 \%$ range. The light-curve of GRB 030329 was characterized by numerous bumps and wiggles, and after about 10 days a supernova component also affected the observations. The modeling of these polarization data is beyond the capabilities of any scenario discussed so far, lacking for instance any clear correlation between polarization and light-curve behaviour. Possibly, the observed emission and polarization is therefore due to the superposition of different phenomena that make a proper modeling difficult to achieve. The vanishing radio polarization at late-times might be due to much less ordered than expected magnetic fields and/or Faraday rotation depolarizing the emission at the radio bands.

\subsection{GRB 121024A}

GRB 121024A was intensively observed with the VLT (Wiersema et al. 2014) starting from a few hours after the GRB and obtaining a positive and highly unexpected detection of circular polarization, $P_{\text {circ }}=0.61 \pm 0.13 \%$, together with an extensive linear polarimetric monitoring. The first observations showed a rather high polarization level, $P_{\text {lin }} \sim 5 \%$, with a global decreasing trend with time and a constant position angle. Observations carried out the night after, showed a lower polarization level with a clear $90^{\circ}$ rotation of the position angle. Analysis of the light-curve allowed to identify a jetbreak between the two sets of observations and this is a very clear identification of the 
polarization angle swing predicted to occur around the jet-break time of a homogeneous jet that is not spreading sideways (Rossi et al. 2004). During the circular polarimetry measurement the linear polarization was about $4 \%$, and therefore the circular to linear polarimetry ratio turned out to be $P_{\text {circ }} / P_{\text {lin }} \sim 0.15$, a very high value, order of magnitudes greater than the theoretical expectations (Toma et al. 2008). If the emission process is synchrotron the expected polarization is indeed $P_{\text {circ }} \sim \gamma_{\mathrm{e}}^{-1}$, where $\gamma_{\mathrm{e}}$ is the random Lorentz factor of the accelerated electrons emitting the observed radiation. This relation holds under the assumption of isotropic pitch-angle distribution and ordered magnetic fields (Toma et al. 2008), and the high value of measured circular polarization poses a challenge to this assumption. Furthermore, a detailed analysis carried out by Nava et al. (2016) suggests that under the hypothesis of optically thin synchrotron emission such a high value of circular to linear polarization ratio is not possible even with extremely isotropic pitch-angle distribution. A satisfactory interpretation of this striking result is still missing.

\section{Conclusions and Perspectives}

The large set of observations available for the afterglows, mainly but not only in the optical, allows us to derive a few important conclusions. First of all, the simple observation of variable polarization implies that the afterglow radiation is intrinsically polarized, thus offering one of the strongest observational evidence for the synchrotron origin of the afterglow emission. The observations of specific patterns (i.e. the position angle swing) during the evolution of the afterglows in polarimetry that have been predicted in advance give also confidence to the general interpretative scenario, although exceptions are present. And the detection of circular polarimetry at a level much higher than expected instead poses a formidable challenge to our present GRB afterglow emission interpretation.

The success of recent observational campaigns clearly shows that a massive approach, trying to follow the afterglow evolution from the early-time, with intermediate-size robotic telescope, to the late phases, with the biggest available facilities, is required. And the parameter space for discoveries is still huge. Radio observations are promising, in particular with future high-sensitivity facilities, and mm observations with ALMA can help to dramatically extend the energy range of the observations and the testing capabilities of the various interpretative scenarios.

For the prompt phase the situation is less clear, but also offering perspective for exciting discoveries in the near future. A final answer to distinguish between intrinsic and geometric models could be obtained by accumulating more observations. Indeed, models (a-b, f) - as defined in Section 2 - predict a polarized emission for all bursts, whereas models (c-e) would predict that only a small fraction of GRBs are highly polarized. This shows the importance of accumulating polarimetric measurements for the understanding of intrinsic properties of GRBs, but the current instrumentation is statistically limited and can provide measurements just for the brightest events.

Recently, a few polarization measurements of the very early optical afterglow have been reported in some cases while the prompt high-energy phase was still on going . The most intruguing case is GRB $120308 \mathrm{~A}$ for which a high level, $\Pi=28 \pm 4 \%$, of linear optical polarization in the early afterglow has been reported by Mundell et al. (2013). The latter measure allowed to point out the presence of a magnetized reverse shock with an ordered magnetic field, confirming the presence of high magnetic fields in the GRB ejecta, and indicating that the multi-wavelength approach could be fruitful, even if there is currently no consensus on the common origin of the $\gamma$-ray and optical emission in the 
prompt phase of GRBs (e.g. Vestrand et al. 2005, Stratta et al. 2009, Götz et al. 2011, Guidorzi et al. 2011, Kopač et al. 2013).

\section{Acknowledgements}

This work has been supported by ASI grant I/004/11/2, by the French Space Agency (CNES) and by the UnivEarthS Labex program at Sorbonne Paris Cité (ANR-10-LABX0023 and ANR-11-IDEX-0005-02).

\section{References}

Bersier, D., et al. 2003, ApJL, 583, L63

Coburn, W. \& Boggs, S. E. 2003, Nature, 423, 415

Covino, S., et al. 1999, A\& $A, 348, \mathrm{~L} 1$

Covino, S., et al. 2003, A\&BA, 400, L9

Covino, S. \& Götz, D. 2016, Astronomical and Astrophysical Transactions, 29, 205

Ghisellini, G. \& Lazzati, D. 1999, MNRAS, 309, L7

McGlynn, S., et al. 2007, A\&SA, 466, 895

Götz, D., et al. 2009, ApJL, 695, L208

Götz, D., et al. 2011, MNRAS, 413, 2173

Götz, D., et al. 2014, MNRAS, 695, L208

Götz, D., et al. 2014, MNRAS, 444, 2766

Granot J. 2003, MNRAS, 596, L17

Granot, J. \& Königl, A. 2003, ApJL, 594, L83

Granot, J. \& Taylor, G. B. 2005, ApJ, 625, 263

Gruzinov, A. \& Waxman, E. 1999, ApJ, 511, 852

Guidorzi, C., et al. 2011, MNRAS, 417, 2124

Harrison, F. A., et al. 1999, ApJL, 523, L121

Israel, G. L., et al. 1999, A\& A, 348, L5

Kopač, et al. 2013, ApJ, 772, 73

Lazzati, D., et al. 2004, MNRAS, 347, L1

Lazzati, D. \& Begelman, M. C. 2009, ApJL, 700, L141

Loeb, A. \& Perna, R. 1998, ApJ, 495, 597

Lyutikov, M., et al. 2003, ApJ, 597, 998

Masetti, N., et al. 2003, A\&SA, 404, 465

Metzger, B. D., et al. 2011, MNRAS, 413, 2031

Mundell, C. G., et al. 2013, Nature, 504, 119

Nakar, E., Piran, T., \& Waxman, E. 2003, JCAP, 10, 005

Nava, L., et al. 2016, MNRAS, 455, 1594

Rossi, E. M., et al. 2004, MNRAS, 354, 86

Rutledge, R. E., Fox, D. B. 2004, MNRAS, 350, 1288

Sari, R. 1999, ApJL, 524, L43

Stanek, K. Z., et al. 1999, ApJ, 522, L39

Stratta, G., et al. 2009, A\&A 503,783

Toma, K., et al. 2008, ApJ, 673, L123

Vestrand, W. T., et al. 2005, Nature, 435, 178

Wang, L. \& Wheeler, J. C. 2008, ARAA, 46, 433

Waxman, E. 2003, Nature, 423, 388

Wiersema, K., et al. 2014, Nature, 509, 201

Wijers, R. A. M. J., et al. 1999, ApJL, 523, L33

Wigger, C., et al. 2004, ApJ, 613, 1088

Yonetoku, D., et al. 2011, ApJL, 743, L30

Yonetoku, D., et al. 2012, ApJL, 758, L1 